\title{
Identification of a novel class of mammalian phosphoinositol-specific phospholipase $C$ enzymes
}

\author{
ALAN J. STEWART ${ }^{1}$, JOY MUKHERJEE ${ }^{2}$, SCOTT J. ROBERTS ${ }^{1}$, \\ DOUGLAS LESTER $^{2}$ and COLIN FARQUHARSON ${ }^{1}$
}

\author{
${ }^{1}$ Division of Gene Function and Development, Roslin Institute, Roslin, Midlothian, EH25 9PS; \\ ${ }^{2}$ Division of Health and Food Sciences, University of Abertay Dundee, Bell Street, Dundee, DD1 1HG, UK
}

Received June 21, 2004; Accepted August 2, 2004

\begin{abstract}
Phosphoinositol (PhoIns)-specific phospholipase C enzymes (PLCs) are central to the inositol lipid signaling pathways and contribute to intracellular $\mathrm{Ca}^{2+}$ release and protein kinase $\mathrm{C}$ activation. Five distinct classes of PhoInsspecific PLCs are known to exist in mammals, which are activated by membrane receptor-mediated events. Here we have identified a sixth class of PhoIns-specific PLC with a novel domain structure, which we have termed PLC- $\eta$. Two putative PLC- $\eta$ enzymes were identified in humans and in mice. Sequence analysis revealed that residues implicated in substrate binding and catalysis from other PhoIns-specific PLCs are conserved in the novel enzymes. PLC- $\eta$ enzymes are most closely related to the PLC- $\delta$ class and share a close evolutionary relationship with other PLC isozymes. EST analysis and RT-PCR data suggest that PLC- $\eta$ enzymes are expressed in several cell types and, by analogy with other mammalian PhoIns-specific PLCs, are likely to be involved in signal transduction pathways.
\end{abstract}

\section{Introduction}

Phospholipase C enzymes (PLCs, EC 3.1.4.3) catalyze the cleavage of membrane phospholipids to 1,2-diacylglycerol (DAG) and their respective phosphoryl compound. In mammals, five distinct classes of PLCs have previously been identified $(\beta, \gamma, \delta, \varepsilon$ and $\zeta)$, all of which specifically react with phosphoinositols (PhoIns) (1-3). PLC-cleavage of phosphatidylinositol (PtdIns) 4,5-bisphosphate results in the generation of DAG and inositol 1,4,5-triphosphate, which promote the activation of protein kinase $\mathrm{C}$ and the release of $\mathrm{Ca}^{2+}$ from intracellular stores, respectively $(4,5)$. The five known classes of mammalian PLCs contain several conserved domains, which include Pleckstrin homology (PH), EF-hand,

Correspondence to: Dr Alan Stewart, Roslin Institute, Roslin, Midlothian EH25 9PS, UK

E-mail: alan.stewart@bbsrc.ac.uk

Key words: $\mathrm{Ca}^{2+}$ signaling, phosphatidylinositol, phospholipase $\mathrm{C}-\eta$, protein kinase $\mathrm{C}$, receptor-mediated signaling, signal transduction catalytic $\mathrm{X}$ and $\mathrm{Y}$ and $\mathrm{C} 2$ domains. The enzymes have been classified on the basis of amino acid sequence and by the mechanisms they activate in response to ligand interactions with various receptors.

PLC- $\beta$ isozymes are activated by the action of $G$ proteins associated with plasma and nuclear membranes (6). G proteins consist of $\alpha, \beta$ and $\gamma$ subunits that are stably bound in the inactive, GDP-bound state. Interface between the G protein and an agonist-occupied receptor triggers the exchange of GDP for GTP on the $\alpha$ subunit and its dissociation from the $\beta$ and $\gamma$ subunits. Both the $\mathrm{G} \alpha$ subunit and $\mathrm{G} \beta \gamma$ dimer activate PLC- $\beta(7,8)$. PLC- $\gamma$ isozymes are activated by the action of a range of receptor protein tyrosine kinases, which autophosphorylate upon binding of various growth factors (9). These phosphorylation sites then function as docking sites for PLC- $\gamma(10)$. The mechanisms of PLC- $\delta$ activation remain poorly understood. PLC- $\delta$ isozymes are more sensitive to $\mathrm{Ca}^{2+}$ than other isozymes and may therefore be sufficient to trigger their activation (1). Another potential regulator of PLC- $\delta$ enzymes is a recently discovered high-molecular-weight G protein (11). This protein forms a complex with PLC- $\delta 1$ in cells stimulated through $\alpha_{1}$-adrenergic or oxytocin receptors $(11,12)$. The PLC- $\varepsilon$ isozymes are involved in Ras signaling. The Ras family of enzymes is a group of GTPases that regulate pathways involved in cell proliferation, differentiation and apoptosis (13). Expression of wild-type PLC- $\varepsilon$ has been shown to promote the generation of the GTP-bound form of Ras in cultured cells $(14,15)$. The last known member of the mammalian PLC family, PLC- $\zeta$, has been identified as the main trigger of $\mathrm{Ca}^{2+}$ oscillations at the very first stage of development, when sperm meets egg (2). PLC- $\delta 1$ also induces $\mathrm{Ca}^{2+}$ oscillations in eggs, albeit less efficiently than PLC- $\zeta$ ( 20-fold higher concentration of PLC- $\delta 1$ is required) (16). This suggests that PLC- $\zeta$ may be activated by a similar mechanism to PLC- $\delta$ isozymes.

PLCs that show specificity toward membrane phospholipids other than PtdIns are present in bacteria (17) and are thought to also exist in mammals. A role in mammalian signal transduction for a phosphocholine (PhoCho)-specific PLC has previously been described $(18,19)$. These studies suggest the existence of other mammalian PLC enzymes. Whilst searching for a mammalian PhoCho-specific PLC, two new PhoIns-specific PLC enzymes were identified. These enzymes, 
which we have named PLC- $\eta 1$ and PLC- $\eta 2$, are described here.

\section{Materials and methods}

Database and sequence analysis. Searches were performed using the Ensembl Genome Browser available from the EMBL and Sanger Institute and with the tBLASTn algorithm (NCBI) (20) using short stretches of sequence (20-30 amino acids) from phosphatidylcholine-specific PLC from Pseudomonas fluorescens (accession no. AJ304443) (17). The Pfam database (available from the Sanger Institute) (21) was used to search for conserved PLC-associated domains in resultant sequences.

Phylogenic analysis. Initial multiple alignments of protein sequences were carried out using the EMBOSS program, EMMA (available from http://www.hgmp.mrc.ac.uk/ Registered/Option/emboss.html). A phylogenic tree was then created from an aligned region (between 141 and 148 amino acids long) using PHYLIP v3.6 (http://evolution.genetics. washington.edu/phylip.html). One hundred bootstrap replicates were initially used to run the analysis. The JonesTaylor-Thornton matrix (22) was then applied to the 100 datasets. A consensus of these datasets was found to give a statistically significant tree with bootstrap values by the Neighbour Joining method (23). The following sequences were used (the hs- and mm- prefixes indicate human and murine forms of the proteins, respectively; accession numbers are shown in parentheses): hsPLC- 31 (Q9NQ66), mmPLC-ß1 (Q9Z1B3), hsPLC-B2 (NP_04564), hsPLC-B3 (NP_000923), mmPLC-B3 (NP_032900), hsPLC-B4 (NP_000924), mmPLC-B4 (NP_038857), hsPLC- $\gamma 1$ (NP_002651), mmPLC- $\gamma 1$ (AAH65091), hsPLC- $\gamma 2$ (P16885), mmPLC- $\gamma 2$ (NP_758489), hsPLC-81 (Q8RB31), mmPLC- $\delta 1$ (Q8R3B1), hsPLC-83 (NP_588614), mmPLC-83 (NP_690023), hsPLC- 84 (NP_116115), mmPLC- 84 (NP_683739), hsPLC-ع, 2303 amino acids form (AAG17145), hsPLC- $\varepsilon, 1994$ amino acids form (AAG28341), mmPLC- $\varepsilon$ (NP_062534), hsPLC- $\zeta$ (NP_149114), mmPLC- $\zeta$ (NP_473407), hsPLC- 11 (XP_042635), mmPLC- $\eta 1$ (ENSMUSP00000047693; mouse NCBIm32), hsPLC- $\eta 2$ (XP_371214), mmPLC- $\eta 2$ (ENSMUSP 00000030929; mouse NCBIm30), hsPLC- $\delta 1$ (P51178).

Semi-quantitative RT-PCR. Total RNAs were extracted from various tissues of male DF1 mice, aged 3-4 weeks, by phenol/chloroform extraction and treated with DNase I (Ambion) according to the manufacturer's instructions. The RT-PCR reaction was carried out using the Qiagen one-step RT-PCR kit. Primer pairs for 18 S RNA were used as a control. The reaction mixture contained $100 \mathrm{ng}$ RNA (25 ng was used with $18 \mathrm{~S}$ RNA primers), $0.6 \mu \mathrm{M}$ primer pairs and $400 \mu \mathrm{M}$ dNTPs. The oligonucleotide primers used were PLC- $\eta$ 1: 5'-GAAAGATGCATGAGTGTAATGC-3' and 5'CTGAAACATTTGCCTGACTTTTC-3'; PLC- $\eta 2$ : 5'CCATCGACTCCATCCAGG-3' and 5'-TCCAGAAAGCGC TGCAGG-3'. These primers sets were designed to span at least one intron so that any amplification from contaminating genomic DNA would be identified. Oligonucleotide primers for 18S RNA were obtained from Ambion. The tissue RNAs

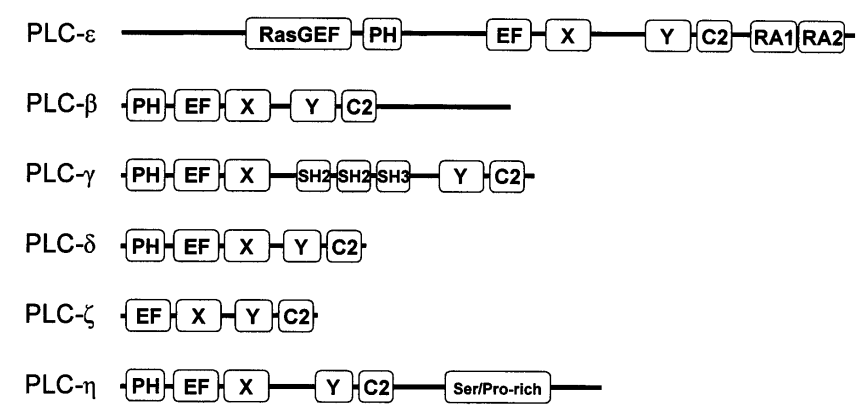

Figure 1. Domain organization in mammalian PhoIns-specific PLC-isozymes. $\mathrm{PH}$, Pleckstrin homology domain; EF, EF-hand domain; X, catalytic X domain; Y, catalytic Y domain, C2, C2 domain; RasGEF, guanine nucleotide exchange factor domain for Ras-like small GTPases; RA, Ras association domain; SH, Src homology domain.

were reverse transcribed for $30 \mathrm{~min}$ at $50^{\circ} \mathrm{C}$, then murine PLC $-\eta 1$ and PLC- $\eta 2$ transcripts were amplified using the cycling profile: $30 \mathrm{sec}$ at $94^{\circ} \mathrm{C}\left(1 \mathrm{st}\right.$ cycle $15 \mathrm{~min}$ at $95^{\circ} \mathrm{C}$ ); $30 \mathrm{sec}$ at $57^{\circ} \mathrm{C} ; 1 \mathrm{~min}$ at $72^{\circ} \mathrm{C}$ with 30 cycles. Each reaction $(12 \mu \mathrm{l})$ was analyzed on $1.5 \%$ agarose gels run in the presence of ethidium bromide $(250 \mu \mathrm{g} / \mathrm{l})$.

\section{Results and Discussion}

Identification of novel PLC enzymes. A search of the human EST database (NCBI) with sequence segments from PhoChospecific PLC from Pseudomonas fluorescens identified two novel genes encoding predicted PhoIns-specific PLCs. The corresponding proteins share $74 \%$ homology and are most closely related to the PLC- $\delta$ class of enzymes, with which they share $\sim 35 \%$ identity. A search within the Pfam database revealed that both enzymes have a novel domain structure containing PH, EF-hand, catalytic $\mathrm{X}$ and $\mathrm{Y}$ and $\mathrm{C} 2$ domains. The two novel enzymes were named PLC- $\eta 1$ and PLC- $\eta 2$ in accordance with current nomenclature. Fig. 1 shows the domain organization within all known mammalian PhoInsspecific PLC isozymes.

The PH domain (absent in PLC- $\zeta$ ) is a module found in many signaling proteins that binds to polyphosphoinositides (24) and indeed, many proteins that contain this domain associate with phospholipid membranes $(25,26)$. However, it has recently been shown that the PH domain of PLC- $\delta 4$ is not essential for membrane localization (27). The C-terminal regions of several $\mathrm{PH}$ domains bind to the $B \gamma$ subunits of $\mathrm{G}$ proteins $(28,29)$. The role of the EF-hand domain in PLCs is unclear, but appears to serve as a flexible link between the $\mathrm{PH}$ and the catalytic domains (30). The active site in PLCs consists of both $\mathrm{X}$ and $\mathrm{Y}$ domains and requires $\mathrm{Ca}^{2+}$ for catalytic function. The $\mathrm{X}$ domain is involved in both substrate and $\mathrm{Ca}^{2+}$ binding, while the $\mathrm{Y}$ domain primarily interacts with the substrate (30). It is these regions that contain the highest degree of sequence similarity among different mammalian PLCs (31). The C2 domain is essential for catalytic activity (32) and often associated with proteins that interact with phospholipids. In some PLCs, the C2 domain binds $\mathrm{Ca}^{2+}$ and mediates $\mathrm{Ca}^{2+}$-dependent interactions with the lipid membrane. It has been speculated that the $\mathrm{C} 2$ domain of PLC- 81 may contain as many as four $\mathrm{Ca}^{2+}$-binding sites (32). 

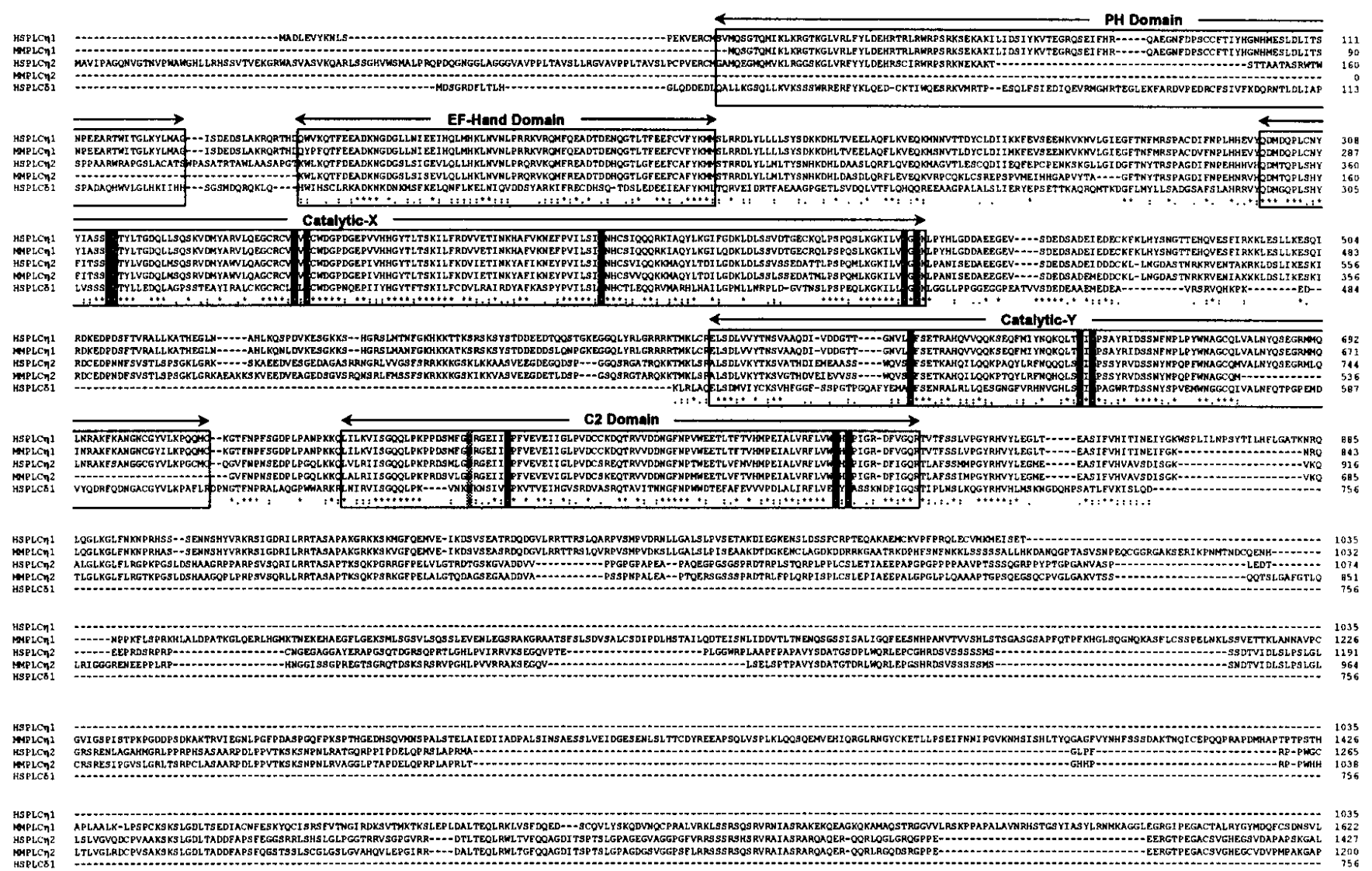

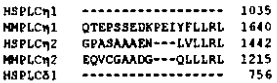

Figure 2. Sequence alignment of human and murine PLC- $\eta$ enzymes with human PLC- $\delta 1$. Conserved and semi-conserved residues implicated in substrate and $\mathrm{Ca}^{2+}$ binding are highlighted (conserved, black boxes; semi-conserved, grey boxes). The following sequences are used (accession numbers are shown in parentheses): HSPLC $\eta 1$, human PLC- $\eta 1$ (XP_042635); MMPLC $\eta 1$, murine PLC- $\eta 1$ (ENSMUSP00000047693); HSPLC 2 , human PLC- $\eta 2$ (XP_371214); MMPLC $\eta 2$, murine PLC- $\eta 2$ (ENSMUSP00000030929); HSPLC 1 1, human PLC-81 (P51178).

The presence of these sequence motifs in PLC- $\eta$ enzymes suggests they may also be involved in signal transduction. In addition to these domains, several PLC isozymes contain other sequence motifs that contribute to their activation. PLC- $\gamma$ isozymes contain SH2 and SH3 domains between the catalytic $\mathrm{X}$ and $\mathrm{Y}$ domains, which facilitate their interaction with receptor tyrosine kinases (10). Whilst PLC- $\varepsilon$ isozymes contain RasGEF and RA domains at their $\mathrm{N}$ - and C-terminal ends, respectively (1).

The genes encoding human PLC- $\eta 1$ and PLC- $\eta 2$ are located on chr.3q25.31 and chr.1p36.32, respectively. Orthologuous proteins were also identified in mice, although it was found that the murine PLC- $\eta 1$ mRNA sequence corresponds to a protein that is $\sim 350$ residues longer at the $\mathrm{C}$-terminal end than its human equivalent. Partial sequences that also appear to be orthologous were found in other species including rat, zebrafish (Danio rerio), puffer fish (Takifugu rubripes) and nematode worm (Caenorhabditis elegans) (data not shown).

The putative PLC- $\eta$ enzymes. Analysis of the full-length human transcripts using the web-based program, SignalP v1.1 (33), predicts the absence of a signal peptide in either protein. This suggests that both proteins are soluble cytoplasmic enzymes. Sequence comparison between PLC- $\eta 1$, PLC- $\eta 2$ and PLC- $\delta s$ reveal significant differences. A sequence alignment of human and murine PLC- $\eta$ isozymes with human PLC- $\delta 1$ is shown in Fig. 2. The region between the $\mathrm{X}$ and $\mathrm{Y}$ catalytic domains is $\sim 100$ residues longer in PLC- $\eta$ enzymes. Also, the human PLC- $\eta$ isozymes contain an additional Cterminal region that is particularly rich in serine and proline residues. Serine- and proline-rich regions have been found in many transcription factors and have a proposed role in proteinprotein interactions $(34,35)$. This region may therefore have functional importance in these enzymes.

The X-ray crystal structure of rat PLC- $\delta 1$ in the presence of bound calcium and inositol 1,4,5-triphosphate reveal that residues His311, Asn312, Glu341, Asp343, and Glu390 coordinate to the catalytic $\mathrm{Ca}^{2+}$ ion. In addition, Lys438, Lys440, Ser522, Arg549 and Tyr551 are shown to specifically interact with inositol 1,4,5-triphosphate (12). This is supported by mutational analysis, which confirm that Asn312, Glu341, Asp343 and Glu390 contribute toward $\mathrm{Ca}^{2+}$-binding and demonstrate Lys438, Ser522 and Arg549 to be important for the preferential hydrolysis of polyphosphoinositides (36). All of the aforementioned PLC- $\delta 1$ residues known to be involved in substrate binding and catalysis are completely conserved within the catalytic X and Y domains of PLC- $\eta 1$ and PLC- $\eta 2$ 


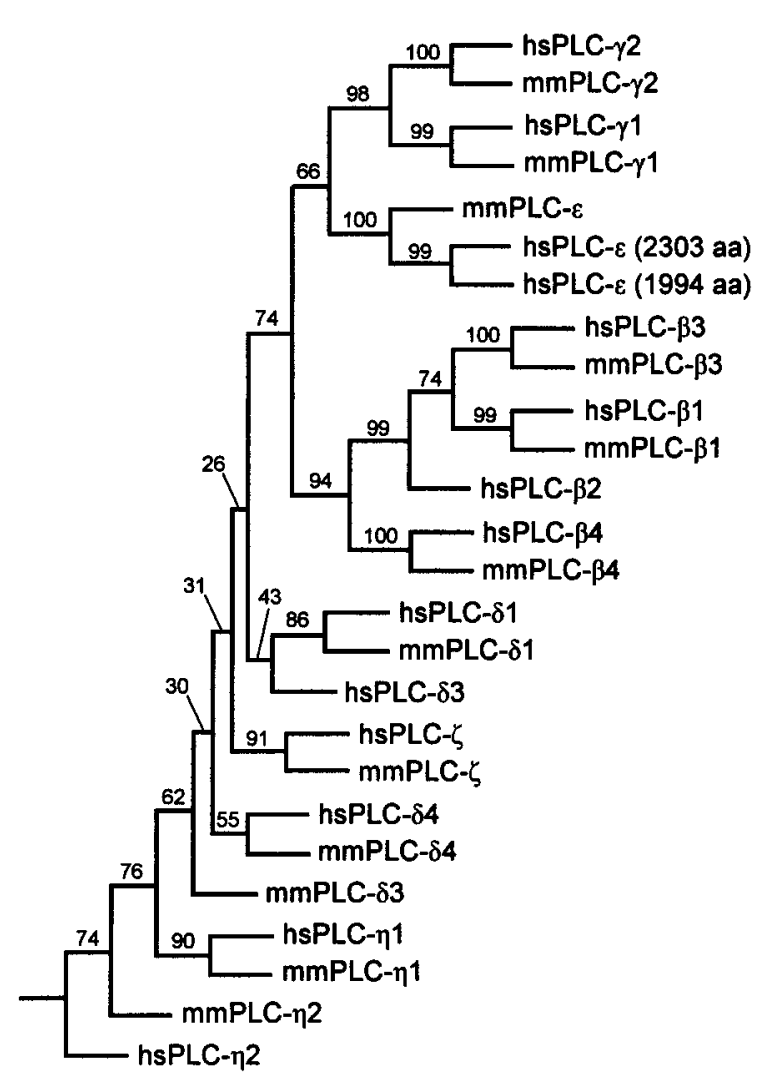

Figure 3. Rooted phylogenic tree of human (hs) and murine (mm) PLCs with bootstrap values inserted at each branch node.

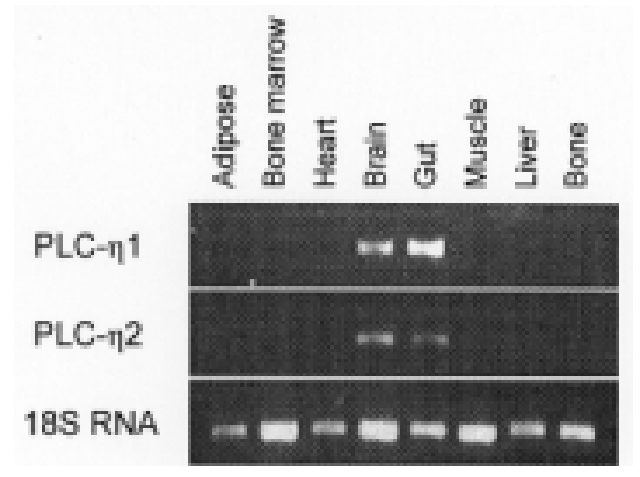

Figure 4. RT-PCR analysis of PLC- $\eta$ enzyme expression in murine tissues. Primers corresponding to 18S RNA were used as a control. The expression of both PLC- $\eta 1$ and PLC- $\eta 2$ enzymes was observed in the brain and gut.

(Fig. 2). As a result, it is very likely that the PLC- $\eta$ enzymes also function as PhoIns-specific phospholipases in vivo.

The $\mathrm{Ca}^{2+}$-mediated lipid-binding site in the $\mathrm{C} 2$ domain of synaptotagmin I consists of four aspartate residues, Asp172, Asp178, Asp230 and Asp232 (37). All four of these residues are conserved in the PLC- $\eta$ enzymes, but not in PLC- $\delta 1$ where Asp172 is equivalent to Asn645 (12). This suggests that $\mathrm{Ca}^{2+}$ ions bind more tightly at this site to synaptotagmin I and to the PLC- $\eta$ enzymes than to PLC- $\delta 1$. Additionally, it indicates that PLC- $\eta$ enzymes, like other PLCs, interact with membrane lipids following $\mathrm{Ca}^{2+}$ binding at this region. Other groups of PhoIns-specific PLCs such as the PLC- $\beta$ and PLC- $\gamma$ enzymes also contain a $\mathrm{C} 2$ domain, yet key residues involved in $\mathrm{Ca}^{2+}$ binding are not conserved (3). This is a similar situation to that observed within some protein kinase $\mathrm{C}$ and synaptotagmin subtypes whose $\mathrm{C} 2$ domains are also unable to bind calcium. It has been speculated that within these enzymes the function of the $\mathrm{C} 2$ domain is to recognize other regulatory lipids or proteins. For example, the $\mathrm{C} 2$ domain of PLC- $\beta 1$ has been found to bind specifically to GTP-charged $\alpha_{\mathrm{q}}$, its physiological activator (38).

Phylogenic analysis performed with human and murine PLC- $\eta 1$ and PLC- $\eta 2$ protein sequences reveal that a close evolutionary relationship exists between these and other PhoIns-specific PLC enzymes. Fig. 3 shows a rooted phylogenic tree of human and murine PLCs with bootstrap values inserted at each branch node. The tree reveals that PLC- $\eta$ isozymes lie closest to the root and supports the classification of these novel enzymes into a new group. PtdIns-specific PLC- $\eta$ enzymes are consequently likely to have formed at an earlier point during evolution than the other PLCs and may exist in a wider range of species.

Tissue distribution of PLC- $\eta$ enzymes. At present, ESTs available in dbEST at the NCBI server indicate that the gene encoding human PLC- $\eta 1$ is expressed in colon, embryonic stem cells, retinoblastoma and teratocarcinoma cells. ESTs corresponding to human PLC- $\eta 2$ were identified in cDNAs isolated from anaplastic oligodendroglioma, epithelioid carcinoma, leukopheresis, lymph, nerve tumor, optic nerve, pancreatic islet, pituitary and retinoblastoma cell populations. Murine ESTs corresponding to PLC- $\eta 1$ were found in cDNAs isolated from brain, eye, kidney, neural retina and upper head. The murine PLC- $\eta 2$ sequence matched ESTs from brain. RT-PCR analysis of RNA isolated from a range of murine tissues revealed bands of expected size corresponding to the expression of both PLC- $\eta$ enzymes in the brain and gut (Fig. 4). The observed expression of both enzymes in the brain by RT-PCR is in agreement with the EST data. No detectable expression was observed in the other tissues assayed.

By analogy with other PhoIns-specific PLCs, these data suggest that the PLC- $\eta$ enzymes may function in the membrane-mediated signaling cascade through intracellular $\mathrm{Ca}^{2+}$ and protein kinase $\mathrm{C}$ pathways in several different cell types. Such pathways are crucial for the initiation of cellular processes including proliferation, differentiation and apoptosis. The discovery of these enzymes is therefore important and is expected to trigger further research towards their cellular function.

\section{Acknowledgements}

We thank the Biotechnology and Biological Sciences Research Council (BBRSC) for funding.

\section{References}

1. Rhee SG: Regulation of phosphoinositide-specific phospholipase C. Annu Rev Biochem 70: 281-312, 2001.

2. Saunders CM, Larman MG, Parrington J, Cox LJ, Royse J, Blayney LM, Swann K and Lai FA: PLC-zeta: a sperm-specific trigger of $\mathrm{Ca}^{2+}$ oscillations in eggs and embryo development. Development 129: 3533-3544, 2002. 
3. Rebecchi MJ and Pentyala SN: Structure, function, and contro of phosphoinositide-specific phospholipase C. Physiol Rev 80: 1291-1335, 2000.

4. Nishizuka Y: The role of protein kinase $\mathrm{C}$ in cell surface signal transduction and tumour promotion. Nature 308: 693-698, 1984.

5. Nishizuka Y: Intracellular signaling by hydrolysis of phospholipids and activation of protein kinase C. Science 258: 607-614, 1992.

6. Divecha $\mathrm{N}$ and Irvine RF: Phospholipid signaling. Cell 80: 269-278, 1995

7. Park D, Jhon DY, Lee CW, Ryu SH and Rhee SG: Removal of the carboxyl-terminal region of phospholipase C-beta 1 by calpain abolishes activation by $\mathrm{G}$ alpha q. J Biol Chem 268: 3710-3714, 1993.

8. Wu D, Jiang H, Katz A and Simon MI: Identification of critical regions on phospholipase $\mathrm{C}$-beta1 required for activation by $\mathrm{G}$ proteins. J Biol Chem 268: 3704-3709, 1993.

9. Kim JW, Sim SS, Kim UH, Nishibe S and Wahl MI: Tyrosine residues in bovine phospholipase C-gamma phosphorylated by the epidermal growth factor receptor in vitro. J Biol Chem 265: 3940-3943, 1990

10. Claesson-Welsh L: Platelet-derived growth factor receptor signals. J Biol Chem 269: 32023-32026, 1994.

11. Feng JF, Rhee SG and Im MJ: Evidence that phospholipase delta1 is the effector in the Gh (transglutaminase II)-mediated signaling. J Biol Chem 271: 16451-16454, 1996.

12. Park ES, Won JH, Han KJ, Suh PG, Ryu SH, Lee HS, Yun HY, Kwon NS and Baek KJ: Phospholipase C-delta1 and oxytocin receptor signalling: evidence of its role as an effector. Biochem J 331: 283-289, 1998.

13. Scita G, Tenca P, Frittoli E, Tocchetti A, Innocenti M, Giardina G and Di Fiore PP: Signaling from Ras to Rac and beyond: not just a matter of GEFs. EMBO J 19: 2393-2398, 2000.

14. Lopez I, Mak E, Ding J, Hamm H and Lomasney JW: A novel bifunctional phospholipase $\mathrm{c}$ that is regulated by Galpha 12 and stimulates the Ras/mitogen-activated protein kinase pathway. J Biol Chem 276: 2758-2765, 2001.

15. Song C, Hu CD, Masago M, Kariyai K, Yamawaki-Kataoka Y, Shibatohge M, Wu D, Satoh T and Kataoka T: Regulation of a novel human phospholipase C, PLC-epsilon, through membrane targeting by Ras. J Biol Chem 276: 2752-2757, 2001.

16. Kouchi Z, Fukami K, Shikano T, Oda S, Nakamura Y, Takenawa T and Miyazaki S: Recombinant phospholipase $\mathrm{C}$-zeta has high $\mathrm{Ca}^{2+}$ sensitivity and induces $\mathrm{Ca}^{2+}$ oscillations in mouse eggs. J Biol Chem 279: 10408-10412, 2004.

17. Preuss I, Kaiser I and Gehring U: Molecular characterization of a phosphatidylcholine-hydrolyzing phospholipase C. Eur J Biochem 268: 5081-5091, 2001

18. Schütze S, Potthoff K, Machleidt T, Berkovic D, Wiegmann K and Krönke M: TNF activates NF-kappa B by phosphatidylcholine-specific phospholipase C-induced 'acidic' sphingomyelin breakdown. Cell 71: 765-776, 1992.

19. Machleidt T, Krämer B, Adam D, Neumann B, Schütze S, Wiegmann $\mathrm{K}$ and Krönke $\mathrm{M}$ : Function of the p55 tumor necrosis factor receptor 'death domain' mediated by phosphatidylcholine-specific phospholipase C. J Exp Med 184: 725-733, 1996.

20. Altschul SF, Madden TL, Schaffer AA, Zhang JH, Zhang Z, Miller W and Lipman DJ: Gapped BLAST and PSI-BLAST: a new generation of protein database search programs. Nucleic Acids Res 25: 3389-3402, 1997.

21. Bateman A, Coin L, Durbin R, Finn RD, Hollich V, Griffiths-Jones S, Khanna A, Marshall M, Moxon S, Sonnhammer ELL, Studholme DJ, Yeats C and Eddy SR: The Pfam protein families database. Nucleic Acids Res 32: D138-D141, 2004
22. Jones DT, Taylor WR and Thornton JM: The rapid generation of mutation data matrices from protein sequences. Comput Appl Biosci 8: 275-282, 1992

23. Saitou $\mathrm{N}$ and Nei $\mathrm{M}$ : The neighbor-joining method: a new method for reconstructing phylogenetic trees. Mol Biol Evol 4: 406-425, 1987.

24. Harlan JE, Hajduk PJ, Yoon HS and Fesik SW: Pleckstrin homology domains bind to phosphatidylinositol-4,5-bisphosphate. Nature 371: 168-170, 1994.

25. Davis LH and Bennett V: Identification of two regions of beta $G$ spectrin that bind to distinct sites in brain membranes. J Biol Chem 269: 4409-4416, 1994.

26. Rebecchi M, Peterson A and McLaughlin S: Phosphoinositidespecific phospholipase C-deltal binds with high affinity to phospholipid vesicles containing phosphatidylinositol 4,5bisphosphate. Biochemistry 31: 12742-12747, 1992.

27. Lee SB, Varnai P, Balla A, Jalink K, Rhee SG and Balla T: The pleckstrin-homology domain of PLC-delta 4 is not critical determinant of the membrane localization of the enzyme. J Biol Chem 279: 24362-24371, 2004.

28. Koch WJ, Inglese J, Stone WC and Lefkowitz RJ: The binding site for the beta gamma subunits of heterotrimeric $G$ proteins on the beta-adrenergic receptor kinase. J Biol Chem 268: 8256-8260, 1993.

29. Touhara K, Inglese J, Pitcher JA, Shaw G and Lefkowitz RJ: Binding of $\mathrm{G}$ protein beta gamma-subunits to pleckstrin homology domains. J Biol Chem 269: 10217-10220, 1994.

30. Essen LO, Perisic O, Cheung R, Katan M and Williams RL: Crystal structure of a mammalian phosphoinositide-specific phospholipase C-delta. Nature 380: 595-602, 1996.

31. Rhee SG and Choi KD: Regulation of inositol phospholipidspecific phospholipase C isozymes. J Biol Chem 267: 12393-12396, 1992.

32. Grobler JA and Hurley JH: Catalysis by phospholipase C-delta1 requires that $\mathrm{Ca}^{2+}$ bind to the catalytic domain, but not the $\mathrm{C} 2$ domain. Biochemistry 37: 5020-5028, 1998.

33. Nielsen H, Engelbrecht J, Brunak S and von Heijne G: Identification of prokaryotic and eukaryotic signal peptides and prediction of their cleavage sites. Protein Eng 10: 1-6, 1997.

34. Ge $\mathrm{H}$ and Roeder RG: Purification, cloning, and characterization of a human coactivator, PC4, that mediates transcriptional activation of class II genes. Cell 78: 513-523, 1994.

35. Kim TK and Roeder RG: Proline-rich activator CTF1 targets the TFIIB assembly step during transcriptional activation. Proc Natl Acad Sci USA 91: 4170-4174, 1994.

36. Ellis MV, James SR, Perisic O, Downes CP, Williams RL and Katan M: Catalytic domain of phosphoinositide-specific phospholipase C (PLC). Mutational analysis of residues within the active site and hydrophobic ridge of PLC-delta1. J Biol Chem 73: 11650-11659, 1998.

37. Sutton RB, Davletov BA, Berghuis AM, Südhof TC and Sprang SR: Structure of the first C2 domain of synaptotagmin I: a novel $\mathrm{Ca}^{2+} /$ phospholipid-binding fold. Cell 80: 929-938, 1995.

38. Wang T, Pentyala S, Elliot J, Dowal L, Gupta E, Rebecchi MJ and Scarlata S: Selective interaction of the $\mathrm{C} 2$ domains of phospholipase C-beta1 and -beta2 with activated Galphaq subunits: an alternative function for $\mathrm{C} 2$-signaling modules. Proc Natl Acad Sci USA 96: 7843-7846, 1999. 